ELECTRONIC RESEARCH ANNOUNCEMENTS OF THE AMERICAN MATHEMATICAL SOCIETY

Volume 3, Pages 114-118 (October 29, 1997)

S $1079-6762(97) 00035-8$

\title{
INVARIANT SETS WITH ZERO MEASURE AND FULL HAUSDORFF DIMENSION
}

\author{
LUIS BARREIRA AND JÖRG SCHMELING
}

(Communicated by Svetlana Katok)

\begin{abstract}
For a subshift of finite type and a fixed Hölder continuous function, the zero measure invariant set of points where the Birkhoff averages do not exist is either empty or carries full Hausdorff dimension. Similar statements hold for conformal repellers and two-dimensional horseshoes, and the set of points where the pointwise dimensions, local entropies, Lyapunov exponents, and Birkhoff averages do not exist simultaneously.
\end{abstract}

\section{INTRODUCTION}

Let $f$ be a continuous map on a compact topological space $X$. For each continuous function $g: X \rightarrow \mathbb{R}$, we define the irregular set for the Birkhoff averages of $g$ by

$$
\mathcal{B}(g)=\left\{x \in X: \lim _{n \rightarrow \infty} \frac{1}{n} \sum_{k=0}^{n} g\left(f^{k} x\right) \text { does not exist }\right\} .
$$

Note that the set $\mathcal{B}(g)$ is $f$-invariant. By Birkhoff's Ergodic Theorem, $\mu(\mathcal{B}(g))=0$ for every $f$-invariant measure $\mu$ on $X$.

The irregular sets $\mathcal{B}(g)$ are usually considered of little interest in ergodic theory and have rarely been considered in the literature. As a rule they are a priori discarded. Here we announce results of [3] showing that in a number of situations ubiquitous in dynamics, the set $\mathcal{B}(g)$ is either empty or carries full topological entropy as well as full Hausdorff dimension.

The results presented here follow from stronger statements proved in [3]. We shall illustrate our methods of proof, which strongly rely on multifractal analysis.

\section{Subshifts OF FINITE TYPE}

Let $\sigma$ be the shift map on $\{1, \ldots, p\}^{\mathbb{N}}$ with the standard topology. Let $A$ be a $p \times p$ matrix whose every entry $a_{i j}$ is either 0 or 1 . Let $\Sigma \subset\{1, \ldots, p\}^{\mathbb{N}}$ be the compact $\sigma$-invariant subset composed of the sequences $\left(i_{0} i_{1} \cdots\right)$ such that $a_{i_{n} i_{n+1}}=1$ for every $n \geq 0$. The map $\sigma \mid \Sigma$ is called the subshift of finite type with transfer matrix $A$. We recall that $\sigma \mid \Sigma$ is topologically mixing if and only if there is a positive

Received by the editors September 2, 1997.

1991 Mathematics Subject Classification. Primary 58F15, 58F11.

Luis Barreira was partially supported by the projects PRAXIS XXI, 2/2.1/MAT/199/94 and JNICT, PBIC/C/MAT/2140/95. Jörg Schmeling was supported by the Leopoldina-Forderpreis.

(c)1997 American Mathematical Society 
integer $k$ such all entries of $A^{k}$ are positive. The topological entropy of $\sigma \mid \Sigma$ is $h(\sigma)=\log \rho(A)$, where $\rho(A)$ denotes the spectral radius of $A$.

We say that two functions $g_{1}$ and $g_{2}$ are cohomologous if $g_{1}-g_{2}=\psi-\psi \circ \sigma+c$, for some $\psi: \Sigma \rightarrow \mathbb{R}$ continuous and $c \in \mathbb{R}$. If $g_{1}$ and $g_{2}$ are cohomologous, then $\mathcal{B}\left(g_{1}\right)=\mathcal{B}\left(g_{2}\right)$. In particular, if $g$ is cohomologous to 0 , then $\mathcal{B}(g)$ is the empty set.

Theorem 1. For a topologically mixing subshift of finite type, the Hölder continuous functions $g_{1}, \ldots, g_{m}$ are non-cohomologous to 0 if and only if

$$
h\left(\sigma \mid \mathcal{B}\left(g_{1}\right) \cap \cdots \cap \mathcal{B}\left(g_{m}\right)\right)=h(\sigma) .
$$

For a topologically mixing subshift of finite type, the family of Hölder continuous functions non-cohomologous to 0 is dense in the space of continuous functions with respect to the supremum norm [3].

Set $\mathcal{B}=\bigcup_{g} \mathcal{B}(g)$. By Theorem $1, h(\sigma \mid \mathcal{B})=h(\sigma)$ for a topologically mixing subshift of finite type. This formula was first established by Pesin and Pitskel' [4] in the case of the Bernoulli shift on two symbols. Their methods of proof are different from ours; moreover, it is not clear if their proof can be generalized to arbitrary subshifts of finite type.

\section{REPELLERS}

Let $f$ be a $C^{1}$ expanding map of a manifold $M$, and $J \subset M$ a repeller of $f$. This means that there are constants $c>0$ and $\beta>1$ such that $\left\|d_{x} f^{n} u\right\| \geq c \beta^{n}\|u\|$ for every $x \in J, u \in T_{x} M$, and $n \geq 1$, and that $J=\bigcap_{n>0} f^{-n} V$ for some open neighborhood $V$ of $J$. The map $f$ is called conformal if $d_{x} f$ is a multiple of an isometry at every point $x \in M$. Examples of conformal expanding maps include one-dimensional Markov maps and holomorphic maps.

Let $\mu$ be a probability measure on $J$. Each Markov partition of a repeller $J$ has associated a one-sided subshift of finite type $\sigma \mid \Sigma$, and a coding map $\chi: \Sigma \rightarrow J$ for the repeller. We define the irregular set for the local entropies of $\mu$ by

$$
\mathcal{H}_{f}(\mu)=\left\{\chi(x) \in J: \lim _{n \rightarrow \infty}-\frac{\log \mu\left(\chi\left(C_{n}(x)\right)\right)}{n} \text { does not exist }\right\},
$$

where $C_{n}(x)$ is the cylinder set of length $n$ containing $x$. We define also the irregular set for the Lyapunov exponents of $f$ by

$$
\mathcal{L}_{f}=\left\{x \in J: \lim _{n \rightarrow \infty} \frac{1}{n} \log \left\|d_{x} f^{n}\right\| \text { does not exist }\right\},
$$

and the irregular set for the pointwise dimensions of $\mu$ by

$$
\mathcal{D}(\mu)=\left\{x \in J: \lim _{r \rightarrow 0} \frac{\log \mu(B(x, r))}{\log r} \text { does not exist }\right\},
$$

where $B(x, r)$ is the ball of radius $r$ centered at $x$. By Kingman's Subadditive Ergodic Theorem, $\mu\left(\mathcal{L}_{f}\right)=0$ for every $f$-invariant measure $\mu$ on $J$. Schmeling and Troubetzkoy [6] proved that $\mu(\mathcal{D}(\mu))=0$ for every measure $\mu$ invariant under an expanding map.

We write $a(x)=\left\|d_{x} f\right\|$ for each $x \in M$, and denote by $\operatorname{dim}_{H} J$ the Hausdorff dimension of $J$. For a repeller $J$ of a conformal $C^{1+\varepsilon}$ expanding map $f$, the equilibrium measure $m_{D}$ of $-\operatorname{dim}_{H} J \cdot \log a$ on $J$ is the unique $f$-invariant measure of maximal dimension. Let $m_{E}$ be the measure of maximal entropy, and $G(f \mid J)$ the family of Gibbs measures on $J$ with a Hölder continuous potential. 
Theorem 2. For a compact repeller of a topologically mixing $C^{1+\varepsilon}$ conformal expanding map, for some $\varepsilon>0$, and $\mu \in G(f \mid J)$, the three measures $\mu, m_{D}$, and $m_{E}$ are distinct if and only if

$$
h\left(f \mid \mathcal{D}(\mu) \cap \mathcal{H}_{f}(\mu) \cap \mathcal{L}_{f}\right)=h(f \mid J) \quad \text { and } \quad \operatorname{dim}_{H}\left(\mathcal{D}(\mu) \cap \mathcal{H}_{f}(\mu) \cap \mathcal{L}_{f}\right)=\operatorname{dim}_{H} J .
$$

\section{HoRSESHOES}

Let $f$ be a $C^{1}$ diffeomorphism of a manifold $M$, and $\Lambda \subset M$ a hyperbolic set for $f$. This means that there is a continuous $d f$-invariant splitting $T_{\Lambda} M=E^{s} \oplus E^{u}$, and constants $c>0$ and $\lambda \in(0,1)$ such that if $x \in \Lambda$ and $n \geq 0$ then $\left\|d_{x} f^{n} v\right\| \leq c \lambda^{n}\|v\|$ for every $v \in E_{x}^{s}$, and $\left\|d_{x} f^{-n} v\right\| \leq c \lambda^{n}\|v\|$ for every $v \in E_{x}^{u}$.

Pesin and we [2], [1] showed that $\mu(\mathcal{D}(\mu))=0$ for every hyperbolic measure $\mu$ invariant under a $C^{1+\varepsilon}$ diffeomorphism. Let $\mathcal{M}_{D}$ be the set of $f$-invariant measures $\mu$ on $\Lambda$ such that $\operatorname{dim}_{H} \mu=\operatorname{dim}_{H} \Lambda$. Note that $\mathcal{M}_{D}$ may be empty.

Theorem 3. For a compact locally maximal saddle-type hyperbolic set for a topologically mixing $C^{1+\varepsilon}$ surface diffeomorphism, for some $\varepsilon>0$, and $\mu \in G(f \mid \Lambda)$, we have $\mu \neq m_{E}$ and $\mu \notin \mathcal{M}_{D}$ if and only if

$$
h\left(f \mid \mathcal{D}(\mu) \cap \mathcal{H}_{f}(\mu)\right)=h(f \mid \Lambda) \quad \text { and } \quad \operatorname{dim}_{H}\left(\mathcal{D}(\mu) \cap \mathcal{H}_{f}(\mu)\right)=\operatorname{dim}_{H} \Lambda .
$$

\section{PROOFS}

The above theorems follow from stronger statements proved in [3]. Here we provide a proof of Theorem 1 in the case $m=1$. It contains all the main ingredients of our methods of proof.

Let $g$ be a Hölder continuous function on $\Sigma$. By the multifractal analysis of Gibbs measures on subshifts of finite type by Pesin and Weiss [5], given $\varepsilon>0$, there are ergodic measures $\mu_{1}$ and $\mu_{2}$ such that $h_{\mu_{i}}(\sigma)>h(\sigma)-\varepsilon$ for $i=1,2$, and

$$
\int_{\Sigma} g d \mu_{1} \neq \int_{\Sigma} g d \mu_{2}
$$

Choose $\delta \in(0, \varepsilon)$ such that $\left|\int_{\Sigma} g d \mu_{1}-\int_{\Sigma} g d \mu_{2}\right|>4 \delta$.

For $i=1,2$ and $\ell \geq 1$, let $\Gamma_{i}^{\ell}$ be the set of points $x \in \Sigma$ such that if $n \geq \ell$ then

$$
\left|\frac{1}{n} \sum_{j=0}^{n} g\left(f^{j} x\right)-\int_{\Sigma} g d \mu_{i}\right|<\delta .
$$

Set $p_{s}=s(\bmod 2)$, and let $\ell_{s}$ be an increasing sequence of positive integers such that $\mu_{p_{s}}\left(\Gamma_{p_{s}}^{\ell_{s}}\right)>1-\varepsilon / 2^{s}$ for each integer $s \geq 1$.

Let $k$ be a positive integer such that all entries of $A^{k}$ are positive, where $A$ is the transfer matrix of $\Sigma$. We define inductively the increasing sequences of positive integers $n_{s}$ and $m_{s}$ by $m_{1}=n_{1}=\ell_{1}$,

$$
m_{s}=\left(n_{s-1}+k+\ell_{s+1}\right) ! \text { and } n_{s}=n_{s-1}+k+m_{s} .
$$

We define families of cylinder sets by $\mathfrak{C}_{s}=\left\{C_{m_{s}}(x): x \in \Gamma_{p_{s}}^{\ell_{s}}\right\}, \mathfrak{D}_{1}=\mathfrak{C}_{1}$, and

$$
\mathfrak{D}_{s}=\left\{\underline{C} C \bar{C}: \underline{C} \in \mathfrak{D}_{s-1}, \bar{C} \in \mathfrak{C}_{s} \text {, and }|C|=k\right\} .
$$

Set

$$
\Lambda=\bigcap_{s \geq 1} \bigcup_{C \in \mathfrak{D}_{s}} C
$$


and define a measure $\mu$ on $\Lambda$ by $\mu(C)=\mu_{1}(C)$ if $C \in \mathfrak{D}_{1}$, and by $\mu(\underline{C} C \bar{C})=$ $\mu(\underline{C}) \mu_{p_{s}}(\bar{C})$ if $\underline{C} C \bar{C} \in \mathfrak{D}_{s}$ for some $s>1$. We extend $\mu$ to $\Sigma$ by $\mu(A)=\mu(A \cap \Lambda)$ for each measurable set $A \subset \Sigma$. If $s>1$ and $\underline{C} \in \mathfrak{D}_{s-1}$, then

$$
\mu\left(\bigcup_{\bar{C} \in \mathfrak{D}_{s}} \underline{C} \cap \bar{C}\right) \geq \mu(\underline{C})\left(1-\frac{\varepsilon}{2^{s}}\right),
$$

and hence, if $\varepsilon<2$ then

$$
\mu(\Lambda) \geq \prod_{s=1}^{\infty}\left(1-\frac{\varepsilon}{2^{s}}\right)>0 .
$$

Let $x \in C \in \mathfrak{D}_{s}$. Note that $\sigma^{|C|-m_{s}} x \in \Gamma_{p_{s}}^{\ell_{s}}$ and $|C| / m_{s} \rightarrow 1$ as $s \rightarrow \infty$. Hence, if $s$ is sufficiently large then

$$
\begin{aligned}
\left|\frac{1}{|C|} \sum_{j=0}^{|C|} g\left(\sigma^{j} x\right)-\int_{\Sigma} g d \mu_{p_{s}}\right| & \\
\leq & \left|\frac{1}{m_{s}} \sum_{j=0}^{m_{s}} g\left(\sigma^{|C|-m_{s}+j} x\right)-\int_{\Sigma} g d \mu_{p_{s}}\right| \cdot \frac{m_{s} \sum_{j=0}^{|C|} g\left(\sigma^{j} x\right)}{|C| \sum_{j=0}^{m_{s}} g\left(\sigma^{|C|-m_{s}+j} x\right)} \\
& +\left|1-\frac{m_{s} \sum_{j=0}^{|C|} g\left(\sigma^{j} x\right)}{|C| \sum_{j=0}^{m_{s}} g\left(\sigma^{|C|-m_{s}+j} x\right)}\right| \cdot\left|\int_{\Sigma} g d \mu_{p_{s}}\right| \\
< & 2 \delta .
\end{aligned}
$$

By the choice of $\delta$, we have $\mathcal{B}(g) \supset \Lambda$.

Now let $x \in \Lambda$. We will prove that if $q$ is sufficiently large then

$$
-\frac{\log \mu\left(C_{q}(x)\right)}{q} \geq h(\sigma)-\eta
$$

for some $\eta \in(0,2 \varepsilon)$. This implies that

$$
h(\sigma \mid \mathcal{B}(g)) \geq h(\sigma \mid \Lambda) \geq h_{\mu \mid \Lambda}(\sigma) \geq h(\sigma)-2 \varepsilon,
$$

and since $\varepsilon$ is arbitrary, $h(\sigma \mid \mathcal{B}(g))=h(\sigma)$.

We proceed by induction on $q$. Choose an integer $s_{q}$ such that $\left|C^{s_{q}}\right| \leq q<$ $\left|C^{s_{q}+1}\right|$, where $\mathfrak{D}_{s_{q}+1} \ni C^{s_{q}+1} \subset C_{q}(x) \subset C^{s_{q}} \in \mathfrak{D}_{s_{q}}$. Assume that

$$
\left|C^{s_{q}}\right| \leq q \leq\left|C^{s_{q}}\right|+k+\ell_{s_{q}+1}
$$

Then $q /\left|C^{s_{q}}\right| \rightarrow 1$ as $q \rightarrow \infty$. By Breiman's Theorem and induction, if $q$ is sufficiently large then

$$
-\frac{\log \mu\left(C_{q}(x)\right)}{q} \geq-\frac{\log \mu\left(C^{s_{q}}\right)}{\left|C^{s_{q}}\right|} \cdot \frac{\left|C^{s_{q}}\right|}{q} \geq h(\sigma)-\eta,
$$

for some $\eta \in(0,2 \varepsilon)$. When (1) does not hold, we have $\mu\left(C_{q}(x)\right)=\mu\left(C^{s_{q}}\right) \mu_{p_{s_{q}+1}}\left(C^{\prime}\right)$, where $C_{q}(x)=C^{s_{q}} C C^{\prime}$ such that $C^{\prime}$ contains an element of $\mathfrak{C}_{s_{q}+1},\left|C^{\prime}\right|>\ell_{s_{q}+1}$, and $|C|=k$. By Breiman's Theorem and induction, if $q$ is sufficiently large then

$$
\begin{aligned}
-\frac{\log \mu\left(C_{q}(x)\right)}{q} & =\left(-\frac{\log \mu\left(C^{s_{q}}\right)}{\left|C^{s_{q}}\right|} \cdot\left|C^{s_{q}}\right|-\frac{\log \mu_{p_{s_{q}+1}}\left(C^{\prime}\right)}{\left|C^{\prime}\right|} \cdot\left|C^{\prime}\right|\right) \frac{1}{\left|C^{s_{q}}\right|+k+\left|C^{\prime}\right|} \\
& \geq h(\sigma)-\eta,
\end{aligned}
$$

for some $\eta \in(0,2 \varepsilon)$. This completes the proof. 


\section{REFERENCES}

1. L. Barreira, Ya. Pesin, and J. Schmeling, On the pointwise dimension of hyperbolic measures: a proof of the Eckmann-Ruelle conjecture, Electron. Res. Announc. Amer. Math. Soc. 2 (1996), no. 1, 69-72. MR 97i:58094

2. L. Barreira, Ya. Pesin, and J. Schmeling, Dimension of hyperbolic measures - a proof of the Eckmann-Ruelle conjecture, WIAS Preprint 245 and IST Preprint 26/96, 1996 (submitted for publication).

3. L. Barreira and J. Schmeling, Sets of "non-typical" points have full topological entropy and full Hausdorff dimension, IST Preprint 14/97, 1997 (submitted for publication).

4. Ya. Pesin and B. Pitskel', Topological pressure and the variational principle for noncompact sets, Functional Anal. Appl. 18 (1984), no. 4, 307-318. MR 86i:28031

5. Ya. Pesin and H. Weiss, A multifractal analysis of Gibbs measures for conformal expanding maps and Markov Moran geometric constructions, J. Statist. Phys. 86 (1997), no. 1/2, 233275. CMP 97:08

6. J. Schmeling and S. Troubetzkoy, Pointwise dimension for regular hyperbolic measures for endomorphisms, in preparation.

Departamento de Matemática, Instituto Superior Técnico, 1096 Lisboa, Portugal

E-mail address: barreira@math.ist.utl.pt

URL: http://www.math.ist.utl.pt/ barreira/

Fachbereich Mathematik und Informatik, Freie Universität Berlin, Arnimallee 2-6, D-14195 Berlin, Germany

E-mail address: shmeling@math.fu-berlin.de 